\title{
EFECTO DE UNA FORMULACIÓN DE CULTIVOS ANDINOS EN UN MODELO MURINO
}

\section{Effects of an andean grain formulation in a murine model}

(iD) Maribel Huatuco Lozano'

'Universidad Le Cordon Bleu, Perú.

2Universidad Nacional Agraria La Molina, Perú.

${ }^{3}$ Pontificia Universidad Católica del Perú, Perú.

Correspondencia:

Mag. Maribel Huatuco Lozano

maribel.huatuco@ulcb.edu.pe

\section{RESUMEN}

El presente trabajo tiene como objetivo determinar el efecto de una formulación de pellets a base de cultivos andinos (arracacha, achira, mashua, oca, cañihua y tarwi) en un modelo murino como criterio útil para el diseño de productos alimentarios con valor agregado. teniendo en cuenta la ganancia de peso y talla de roedores de la cepa BALB/C-53. La metodología de Taguchi fue utilizada en el proceso de formulación y optimización, la cual permitió trabajar con seis factores: arracacha (Ar) Arracacia xanthorrhiza, achira (Ac) Canna indica L., mashua (Ma) Tropaeolum tuberosum, oca (Oc) Oxalis tuberosa, cañihua (Ca) Chenopodium pallidiulle aellen, tarwi (Ta) Lupinus mutabilis, con 2 niveles de trabajo, para ello se determinó matriz ortogonal L8 (27). Se formaron 8 grupos de tratamientos para cada una de las formulaciones en forma de pellets (F1, F2, F3, F4, F5, F6, F7 y F8) y un grupo control, se administró durante 15 días las diferentes formulaciones y al grupo control alimento comercial (ratonina). Los resultados obtenidos muestran que la F7 (20\% Ar, 20\% Ac, 15\% Ma, 15\% Oc, 20\% Ca y $20 \%$ de Ta) fue la más significativa en atribuir el peso, seguida de la F8 y F2 mientras que la F2, F7, y F3 fueron las más influyentes en la obtención de talla de los ratones. Por lo tanto, el efecto de las formulaciones de granos andinos más resaltante con respecto a la atribución de peso y talla en un modelo murino fue la F7 seguida por la F2.

Palabras clave: Formulación, granos andinos, modelo murino.

\section{ABSTRACT}

In order to design products with added value and characterized as functional, different formulations of pellets were evaluated based on varieties of arracacha, achira, mashua, oca, cañihua and tarwi. For this purpose, the effect of an Andean grain formulation was evaluated. murine model, considering the gain of weight and size of rodents of the strain BALB / C-53. The methodology of Taguchi was used in the formulation and optimization process, which allowed to work with six factors: arracacha (Ar) Arracacia xanthorrhiza, achira (Ac) Canna indica L., mashua (Ma) Tropaeolum tuberosum, oca (Oc) Oxalis tuberosa, cañihua (Ca) Chenopodium pallidiulle aellen, tarwi (Ta) Lupinus mutabilis, with 2 work levels, for which 8 experimental runs were determined 
(orthogonal matrix L8 (27). 8 groups of treatments were formed for each of the formulations in the form of pellets that were obtained with the Taguchi method (F1, F2, F3, F4, F5, F6, F7 and F8) and a control group was administered for 15 days. days the formulations and the commercial food control group (ratonine). The obtained results show that the F7 was the most significant in attributing the weight, followed by the F8 and F2 while the F2, F7, and F3 were the most influential in obtaining the size of the mice. Therefore, the effect of the Andean grains formulations, which are the most outstanding with respect to the attribution of weight and height in a murine model, was F7 and followed by F2.

Key words: Formulation, andean grains, murine model.

\section{INTRODUCCIÓN}

Un aspecto importante para toda nación es lograr la seguridad alimentaria, apoyada en la basta biodiversidad que sustentan los diferentes ecosistemas que dispone el Perú. Sin embargo, una parte de esta biodiversidad aún permanece sin estudios que analicen a plenitud su potencial para contribuir con el desarrollo promoviendo su transformación y uso sostenible. Un ejemplo de ello es la biodiversidad en cereales, tubérculos y raíces andinas, cuyos valores nutricionales, y, contenido de sustancias bioactivas no han sido plenamente inventariados y en menor medida aprovechados para la producción y la provisión de la seguridad alimentaria. Esto trae como efecto directo la subutilización de la biodiversidad y consiguientemente acentúa la dependencia del sistema agroalimentario del país (García y Cadima, 2003; Barrera, Tapia, y Monteros, 2004).

Se estima que los cultivos andinos, básicamente cereales, tubérculos y raíces, ocupan una superficie que se extiende desde los 1500 a 3200 msnm, presente en más de diez departamentos, todos ellos ubicados en la sierra, y en zonas de pobreza extrema. Se tienen un total de siete cultivos y, entre 60 y 100 variedades por cultivo, en dependencia de las zonas de divergencia genética, tales como Cusco, Puno, Cajamarca, entre otros (Tapia y Fries, 2007), por su contenido de nutrientes, estos cultivos se pueden dividir en los que aportan: (a) Una cantidad importante de proteínas (cañihua, quinua y amaranto); (b) un elevado contenido de proteínas y grasas (tarwi); principalmente carbohidratos (tubérculos y raíces); (c) buenas cantidades de carotenos, como la achira, arracacha y (d) un buen contenido de minerales como la cañihua (Tapia, 2000).

Estos atributos, diversidad y composición nutricional son de utilidad para diseñar productos alimentarios con valor agregado, que subyace en la determinación del valor nutricional de las diferentes variedades de cereales, tubérculos y raíces andinas. En el ámbito social y económico, contribuyen con políticas tendientes a la lucha contra la desnutrición, a la creación de nuevos puestos de trabajo, generación de ingresos alternativos, y, al fortalecimiento y desarrollo de la gastronomía nacional.

\section{MATERIAL Y METODOS Características del estudio}

El estudio realizado se inscribe en el ámbito de una alcance descriptivo, relacional y experimental. De ese modo, la población y muestra en estudio estuvo conformada por 45 ratones (Muss musculus) macho de 21 días de nacido de la cepa BALB/C-53, seleccionados al azar provenientes del Instituto Nacional de Salud (Alpizar \& Ruiz, 2013). De otro lado, las muestras fueron colectadas de los mercados ubicados en Puente Piedra y La Parada, teniendo en cuenta que la procedencia corresponda a las regiones de Cajamarca, Puno y Cusco quedando registrado en una Ficha. La preparación del alimento consideró en su formulación un (1) kg de cada una de las especies evaluadas, que estuvieron compuestas por arracacha amarilla (Arracacia xanthorrhiza), achira morada (Canna edulis), mashua naranja amarillo (Tropaeolum tuberosum), oca amarilla (Oxalis tuberosa), cañihua (Chenopodium pallidicaule) y tarwi (Lupinus mutabilis). 


\section{Formulaciones}

Las formulaciones consideraron el método señalado por Taguchi, Elsayed, y Hsiang (1989), que tiene por objeto la optimización de productos y procesos, a fin de asegurar productos robustos, de alta calidad y bajo costo para ello desarrolló una serie de arreglos particulares conforme a la siguiente expresión:

$$
L_{a}(b)^{c}
$$

Donde, a: Representa el número de pruebas o condiciones experimentales que se tomarán. Esto es el número de renglones o líneas en el arreglo; b: representa los diferentes niveles a los que se tomará cada factor y c: es el número de efectos independientes que se pueden analizar, esto es el número de columnas. De ese modo, se determinó los factores o ingredientes influyentes en la formulación de productos que permitió obtener una combinación óptima de diseño de parámetros para que el producto sea funcional y con un alto nivel de calidad de los productos elaborados.

Las variables independientes, en el estudio, fueron seis: arracacha (Ar) Arracacia Xanthorrhiza, achira (Ac) Canna indica L., mashua (Ma) Tropaeolum tuberosum, oca (Oc) Oxalis tuberosa, cañihua (Ca) Chenopodium pallidiulle aellen, tarwi (Ta) Lupinus mutabilis, con dos niveles (Tabla 1) y como variable dependiente la formulación (F) donde se determinó ocho (8) corridas experimentales (matriz ortogonal L8 $\left(2^{7}\right)$ ). Las variables por analizar fueron comparación nutricional (CN), y señal a ruido (S/R).

\begin{tabular}{lcc}
$\begin{array}{l}\text { Tabla } 1 \\
\text { Niveles considerados } \\
\text { Taguchi }\end{array}$ & en diseño & experimental \\
\hline $\begin{array}{l}\text { Factores de } \\
\text { control }\end{array}$ & 1 & 2 \\
\hline Arracacha (Ar) & 15 & 20 \\
Achira (Ac) & 15 & 20 \\
Mashua (Ma) & 15 & 20 \\
Oca (Oc) & 15 & 20 \\
Cañihua (Ca) & 15 & 20 \\
Tarwi (Ta) & 15 & 20 \\
\hline
\end{tabular}

Nota: $F$ *1: mínimo; 2: máximo

En la Tabla 2, se muestran las ocho (8) del control (Ar, Ac, Ma, Oc, Ca y Ta), y donde formulaciones experimentales, determinadas las variables a analizar son la comparación por la matriz ortogonal $L_{8}\left(2^{7}\right)$, que reflejan nutricional $(C N)$, y señal a ruido $(S / R)$. las diferentes combinaciones de los factores 
Tabla 2

\section{Formulaciones experimentales con diseño de Taguchi}

\begin{tabular}{lllllll}
\hline $\begin{array}{l}\text { Punto } \\
\text { de }\end{array}$ & Ar & Ac & Ma & Oc & Ca & Ta \\
diseño & & & & & & \\
\hline F1 & 1 & 1 & 1 & 1 & 1 & 1 \\
F2 & 1 & 1 & 1 & 2 & 2 & 2 \\
F3 & 1 & 2 & 2 & 1 & 1 & 2 \\
F4 & 1 & 2 & 2 & 2 & 2 & 1 \\
F5 & 2 & 1 & 2 & 1 & 2 & 1 \\
F6 & 2 & 1 & 2 & 2 & 1 & 2 \\
F7 & 2 & 2 & 1 & 1 & 2 & 2 \\
F8 & 2 & 2 & 1 & 2 & 1 & 1
\end{tabular}

En la Tabla 3, se presenta los procedimientos desarrollados en la elaboración de las formulaciones de alimentos en forma de pellet que se administró a los grupos tratados (Mosquera, 2014). Además, debe indicarse que los ratones macho fueron mantenidos bajo condiciones estándar de bioterio (con 22-24
${ }^{\circ} \mathrm{C}$ de temperatura; fotoperíodo de $14 \mathrm{~h}$ luz: 10 h oscuridad), con alimento (ratonina) para el control y para los tratamientos las formulaciones de alimentos en forma de pellets elaborados y agua ad libitum (Fuentes, Mendoza, Rosales, y Cisneros, 2008).

Tabla 3

Procedimiento en la elaboración de las formulaciones de alimentos en forma de pellet

\begin{tabular}{cl}
\hline Orden & \multicolumn{1}{c}{ Procedimiento } \\
\hline 1 & Recepción de la materia prima (Ar, Ac, Ma, Oc, Ca y Ta) \\
2 & Lavado de la materia prima (Ar, Ac, Ma, Oc, Ca y Ta) \\
3 & Pelado de la materia prima (Ar, Ac, Ma, Oc, y Ta) \\
4 & Cocción del tarwi (Ta) \\
5 & Cortado de la materia prima (Ar, Ac, Ma, Oc) \\
6 & Molienda por separado de la materia prima (Ar, Ac, Ma, Oc, Ca y Ta) \\
7 & Pesado con una balanza según como la formulación indica. \\
8 & Mezclado de los 6 alimentos. \\
9 & Peletizado con ayuda de una manga pastelera \\
10 & Secado a estufa a una temperatura de $80^{\circ} \mathrm{C}$ por 50 min. \\
11 & Almacenamiento en envases de plástico previamente rotulados según la \\
& formulación
\end{tabular}




\section{Diseño experimental}

Se formaron ocho (8) grupos de tratamientos para cada una de las formulaciones en forma de pellets que se obtuvieron con el método de Taguchi (F1, F2, F3, F4. F5. F6. F7 y F8) y un grupo control. Se administró desde el día 1 al día 15 las formulaciones de alimentos en forma de pellets a los grupos tratamientos y al grupo control alimento comercial (ratonina) para ello se pesó 5 g para cada grupo según corresponda, el agua en ambos grupos fue ad libitum (Fig. 1). Se procedió a pesar y tallar a los ratones macho el día 1, día 5, día 10 y el día 15 (día de evaluación) a la misma hora, mediante una balanza con 0,01 gr de sensibilidad y con un centímetro; para su identificación fueron marcados con piquetes en las orejas.

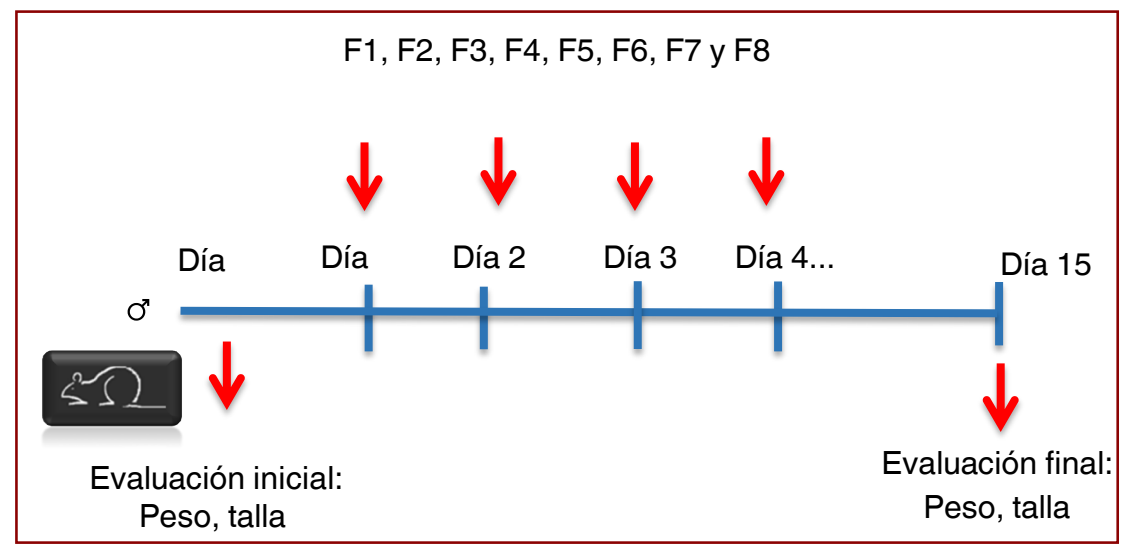

Figura 1. Administración oral de las formulaciones de alimentos en forma de pellets

\section{Instrumentos de recolección de datos, técnicas de procesamiento y análisis}

Los principales instrumentos que se utilizaron fueron: (i) formato de registro de identificación de las variedades de arracacha, achira, mashua, oca, cañihua y tarwi; (ii) equipos e instrumental de laboratorio para la comparación nutricional de las variedades mencionadas anteriormente, teniendo en cuenta la ganancia de peso y el incremento de la talla de los ratones y hoja de identificación de ratones según grupo control y tratamiento. Por último, debe indicarse que se analizaron los datos mediante la utilización del programa Statistical Package for the Social Sciences SPSS ${ }^{\circledast}$ para Windows ${ }^{\circledR}$ versión 24 y Microsoft Office Excel $^{\otimes}$ 2010, realizándose el análisis de varianza (ANOVA) en las variaciones porcentuales para las comparaciones intergrupos, ambas seguidas del post hoc de Tukey con el objetivo de identificar las posibles diferencias. El estudio admitió el valor de $p<0,05$ para la significación estadística.

\section{Aspectos éticos}

En cuanto a los aspectos éticos, se tomó en cuenta lo señalado por el Comité Institucional de Ética para el Uso de Animales (CIEA) del Instituto Nacional de Salud (INS), en el año 2012, que establece que se deben cumplir cuatro principios éticos siendo estos, el respeto, la no maleficencia, la justicia, así como la gratitud y afecto (Fuentes, Mendoza, Rosales, y Cisneros, 2008; Instituto Nacional de Salud, 2012). Bajo ese contexto, la investigación es éticamente aceptable, debido a que sigue el principio de las tres $\mathrm{R}$ de la experimentación humanizada para con los animales, propuesta por William Russell y Rex Burch (1959): i) Reducir, al máximo el número de ellos y, por ende, el total de animales utilizados en investigación; ii) Reemplazar, siempre que sea posible el animal de experimentación por otro modelo experimental, cuando no resulte imprescindible el uso de animales; iii) Refinar, los métodos y técnicas utilizados de modo que produzcan al animal el menor sufrimiento posible (Pérez, 2007). 
En la presente investigación y en cumplimiento con los principios éticos establecidos por INS, los animales de experimentación fueron mantenidos sin ocasionar molestias, maltratos, distrés y dolor; las condiciones de vida fueron apropiadas a su especie y contribuyeron a su salud y confort, así mismo se contó con un profesional entrenado en el manejo de animales de experimentación.

\section{RESULTADOS}

\section{Ganancia de peso de los ratones}

El peso corporal promedio de los nueve grupos de ratones, tratados durante el experimento, en relación con las formulaciones de la dieta que recibieron al día 05 mostró un incremento para las formulaciones F2 y F7, en el día 10, para las formulaciones F3 y F8 y en el día 15 para la F7; mientras que la ganancia de peso con respecto al primer día y el último día de tratamiento se observó en tres grupos de ratones con las formulaciones F7 seguido de la F2 y F3 mientras que la pérdida de peso se evidenció en la formulación F6 (Figura 2). La Tabla 4 muestra los resultados del análisis de varianza (ANOVA), según el cual se aprecian diferencias significativas entre las formulaciones con relación al peso de los ratones $(p<0,05)$.

\section{Tabla 4}

Análisis ANOVA respecto al peso de los ratones

\begin{tabular}{lccccc}
\hline $\begin{array}{l}\text { Fuentes de } \\
\text { variación }\end{array}$ & $\begin{array}{c}\text { Suma de } \\
\text { cuadrados }\end{array}$ & gl & $\begin{array}{c}\text { Media } \\
\text { cuadrática }\end{array}$ & $\mathrm{F}$ & Sig. \\
\hline Entre grupos & 71,323 & 8 & 8,915 & 4,023 &, 002 \\
Dentro de grupos & 79,777 & 36 & 2,216 & & \\
Total & 151,100 & 44 & & & \\
\hline
\end{tabular}

Para determinar las formulaciones más influyentes en obtención de peso según el tipo de formulación se realizó la prueba de Tukey, tal como se aprecia en la Tabla 5, la formulación 7 fue la más influyente, seguida de la formulación 2 y 8

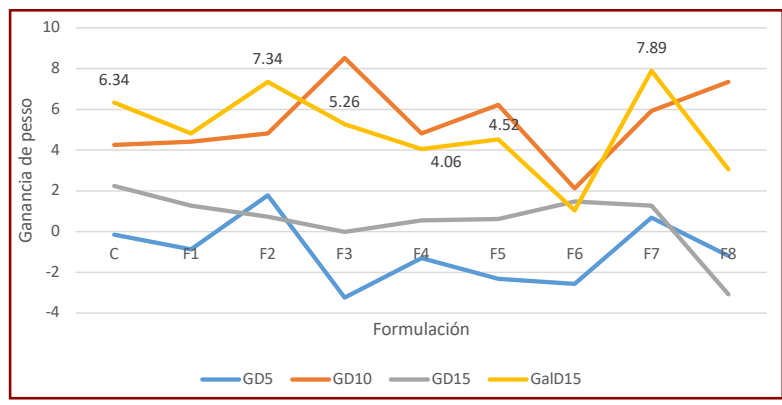

Figura 2. Ganancia de peso según formulaciones 
Tabla 5

Determinación de la formulación que influye en el peso de los ratones-Prueba de Tukey

\begin{tabular}{|c|c|c|c|}
\hline \multirow{2}{*}{ Formulación } & \multirow[b]{2}{*}{$\mathrm{N}$} & \multicolumn{2}{|c|}{ Subconjunto para alfa $=0.05$} \\
\hline & & 1 & 2 \\
\hline Grupo control & 5 & 23.976 & \\
\hline Formulación 5 & 5 & 23.994 & \\
\hline Formulación 6 & 5 & 25.718 & 25.718 \\
\hline Formulación 4 & 5 & 25.736 & 25.736 \\
\hline Formulación 1 & 5 & 25.823 & 25.823 \\
\hline Formulación 3 & 5 & 26.294 & 26.294 \\
\hline Formulación 8 & 5 & 26.928 & 26.928 \\
\hline Formulación 2 & 5 & & 27.204 \\
\hline Formulación 7 & 5 & & 27.894 \\
\hline Sig. & & .073 & .362 \\
\hline
\end{tabular}

\section{Incremento de Talla en los ratones}

La talla corporal promedio de los nueve grupos de ratones con relación con las formulaciones de la dieta que recibieron al día 15, mostró un incremento para las formulaciones F7, F2 y F3 (Figura 3). En la Tabla 6, se observa que las diferencias de tallas encontradas en los ratones al administrar las formulaciones en estudio mostraron ser significativas estadísticamente al aplicar el ANOVA $(p<0,05)$.

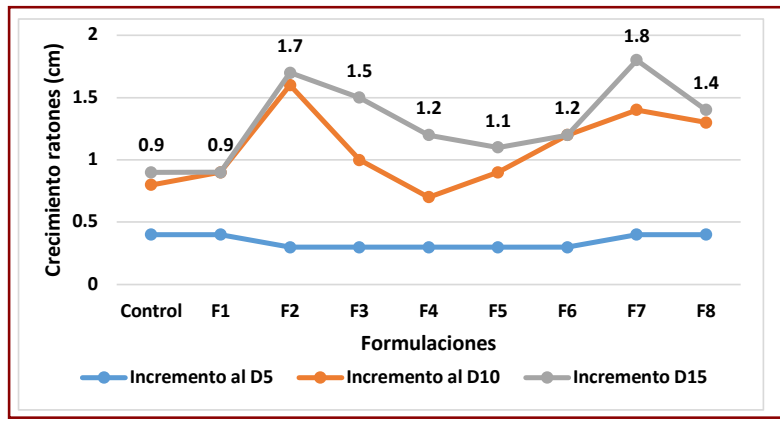

Figura 3. Incremento de talla según formulaciones 
Tabla 6

Análisis de ANOVA respecto a la talla de los ratones

\begin{tabular}{lccccc}
\hline & $\begin{array}{c}\text { Suma de } \\
\text { cuadrados }\end{array}$ & gl & Media cuadrática & F & Sig. \\
\hline Entre grupos & 15,702 & 8 & 1,963 & 8,769 &, 000 \\
Dentro de grupos & 8,058 & 36 &, 224 & & \\
Total & 23,760 & 44 & & & \\
\hline
\end{tabular}

Al igual que el caso anterior se utilizó la prueba de Tukey para determinar las formulaciones más influyentes en la talla según el tipo de formulación, como se aprecia en la Tabla 7, la formulación F2,
F7, y F3 fueron las más influyentes; sin embargo, la menos influyente resultó la formulación 5 en la talla al ratón.

Tabla 7

Determinación de la formulación que influye en la talla de los ratones-Prueba de Tukey

\begin{tabular}{|c|c|c|c|c|}
\hline \multirow{2}{*}{ Formulación } & \multirow[b]{2}{*}{$\mathrm{N}$} & \multicolumn{3}{|c|}{ Subconjunto para alfa $=0.05$} \\
\hline & & 1 & 2 & 3 \\
\hline Grupo control & 5 & 17.611 & & \\
\hline Formulación 5 & 5 & 17.692 & & \\
\hline Formulación 6 & 5 & 17.818 & & \\
\hline Formulación 1 & 5 & 17.881 & 17.881 & \\
\hline Formulación 4 & 5 & 18.266 & 18.266 & 18.266 \\
\hline Formulación 8 & 5 & & 18.842 & 18.842 \\
\hline Formulación 3 & 5 & & & 19.016 \\
\hline Formulación 7 & 5 & & & 19.044 \\
\hline Formulación 2 & 5 & & & 19.046 \\
\hline Sig. & & .434 & .061 & .219 \\
\hline
\end{tabular}

\section{DISCUSIÓN}

Actualmente, no se disponen de estudios relacionados con el efecto de formulaciones de cultivos andinos, en un modelo murino, previo para el diseño de productos con valor agregado, por su alto contenido de macronutrientes, minerales, vitaminas, fibra y especialmente proteínas (Apaza-Moroco, Hayqui-Betancur, \& Sumire-Qquenta, 2015; Jacobsen y Mujica, 2006; León y Urbina, 2015; Matos-Chamorro y MuñozAlegre, 2010; Ministerio de Salud, 2017). En ese sentido, se determinó el efecto de las diferentes formulaciones obtenidas por el método Taguchi, teniendo en cuenta la ganancia de peso y talla en los roedores.

Se estableció que la ganancia del peso corporal promedio de los nueve grupos de ratones, tratados durante el experimento, en relación con las formulaciones de la dieta que recibieron, las cuales mostraron que tres grupos de ratones 
ganaron peso siendo las formulaciones F7 y F2 las que presentaron en común en su composición $20 \%$ de tarwi y cañihua y un 15 $\%$ de mashua a diferencia de la F3 que solo presentó en común 20\% de tarwi; por lo cual se puede indicar que dichos resultados se deben al alto valor proteico que presenta el tarwi (Carvajal-Larenas, Linnemann, Nout, Koziol, y van Boekel, 2016; Ministerio de Salud, 2017; ChirinosArias, 2015) y presenta alto contenido de fósforo, potasio y hierro (Ortega-David, Rodriguez, David, y Zamora-Burbano, 2010), así como vitaminas y minerales (Castañeda, Manrique, Gamarra, Muñoz, y Lizaraso, 2008), así como la cañihua (Gallego-Villa, Russo, Kerbab, Landi, y Rastrelli, 2014; Ministerio de Salud, 2017) y en menor proporción la mashua.

En otros estudios, se encontraron diferencias significativas $(p<0.05)$ en la ganancia de pesoentre las ratas alimentadas con las galletas de granos andinos (quinua, cañihua y tarwi) en relación con el grupo control (trigo) y se concluye, que el contenido de proteínas y nutrientes de los granos andinos se complementan mejor con la harina de trigo y el consumo de galletas formuladas con estas mezclas podrían tener efecto positivo sobre la ganancia de peso (Apaza, 2019). Así mismo, en otra investigación se utilizaron ratas para determinar el crecimiento y la conversión alimenticia empleando el pejibaye (Bactris gasipaes), se suministraron extractos acuosos de pulpa, cascara, semilla y pejibaye entero tanto crudo como cocido, el cual se dio como agua durante seis (6) semanas consecutivas a grupos compuestos también por de seis (6) ratas, en el cual se obtuvo que el grupo que consumió el pejibaye entero cocido presentó mayor aumento de peso. Este efecto, se acentuó aún más en las siguientes semanas donde la diferencia con el resto de los grupos fue más notoria, la cual podría atribuirse a la inactivación de los Factores Antinutricionales durante el proceso de cocción. Los grupos que presentaron pesos inferiores fueron los que consumieron extracto de cascara y semilla de pejibay (Gómez, Vargas, y Quesada, 1998).

En investigaciones realizadas para evaluar el efecto de la ingesta de dietas con distinto contenido de fibra en el peso y grasa corporal en ratones de la cepa BALB/c, inducidos a obesidad, obtuvieron un menor incremento de peso en los grupos de ratones que ingirieron mayor cantidad de fibra dietaría, aunque esta diferencia no fue estadísticamente significativa ( $p$-valor =0,363) (Martínez y Matallana, 2013). De forma similar, se puede comparar con los resultados obtenidos por Cedeño (2013), quien formulo y elaboro dos alimentos balanceados con requerimientos nutricionales apropiados y con base en ingredientes naturales para ratones de experimentación (Mus musculus) determinando que la F1 es óptima para el crecimiento y mantenimiento de los ratones en 34 días de ensayo.

Por su parte, Torres (2003), realizó un estudio en un periodo de 14 días para determinar el efecto de administración de dosis crecientes de la formulación sobre el peso vivo en ratones determinando que si existe una cierta tendencia a la elevación del incremento promedio de peso con respecto al tratamiento control; sin embargo, se presentan tratamientos irregulares en los días de estudio por lo que dicho autor no puede definir un claro patrón de elevación sugiriendo que se prolongue los días de evaluación a 20 días. Otros estudios que, también evaluaron ganancia de peso además de la calidad proteica y digestibilidad, en ocho dietas compuestas con base en dos leguminosas, en ratas Wistar durante cuatro semanas, encontraron diferencias significativas en la ganancia de peso en relación a la dieta control, principalmente en los grupos de ratas que consumieron la dieta D maní 75\% con un valor medio después de las cuatros semanas de $226.00 \mathrm{~g}(\mathrm{SD}=55.29)(\mathrm{p}<0.5)$, dieta $\mathrm{H}$, ajonjolí 75\%, con un valor medio de $218.16 \mathrm{~g}$ $(\mathrm{SD}=56.28)(\mathrm{p}<.05)$ ambas complementadas con leche descremada. Por lo tanto, se concluye que formulaciones basadas en leguminosas podrían tener potencial uso en la nutrición humana (Cuj, Mazariegos, Pérez, Fischer, y Román, 2017). Así mismo, se determinó que existe diferencia significativa entre las formulaciones al dar como resultados el peso de los ratones con un $\mathrm{p}$ value $=0$. Además, mediante la prueba de Tukey 
se determinó que la formulación 7 fue la más influyente en la obtención de peso con 27.8940 $\mathrm{g}$ mientras que el grupo control fue de $23.9770 \mathrm{~g}$. De ese modo, queda demostrado que el alimento comercial no influye en el peso. Sin embargo, en otras investigaciones de enfoque nutricional realizadas sobre una dieta rica en calcio minimiza la adsorción de grasas en ratas se determinó que el aumento de peso fue característico y notable para el grupo con dieta alta en grasa $(p<0.05)$, semana 1 a la última semana de tratamiento, a diferencia del grupo tratado con calcio y grasas, que en la última semana de tratamiento disminuyó su peso corporal, lo que indicó que la absorción de grasas disminuye (Barrientos, Becerra, Flores y Beltrán, 2013).

Respecto a la talla se determinó que, si existe diferencia significativa entre las formulaciones F7, F2 y F3 al dar como resultados la talla de los ratones con un $p$ value inferior al 0.05. Además, mediante la prueba de Tukey se determinó que las formulaciones más influyentes son F7, F2 y F3 en la talla de los ratones, debido a que las proteínas tienen función estructural; por lo tanto, la formación de diversos tejidos del organismo se ven beneficiadas (Cuj, Mazariegos, Pérez, Fischer, y Román, 2017; Márquez-Montes, AltúzarCarpio, Villanueva-Carrillo, y Palacios-Pola, 2010; Olza Meneses, Porres Foulquie, Urbano Valero, Martinez de-Victoria, y Gil Hernandez, 2008), así como el aporte en calcio y fosforo de la achira y cañihua (Morales Ordoñez, 2016; Repo-Carrasco, 2009) incrementándose la talla al finalizar los 15 días de evaluación.

\section{CONCLUSIONES}

El efecto de las formulaciones de los cultivos andinos seleccionados en este trabajado, se evidencia que para los atributos peso y talla aplicado en el modelo murino, la formulación F7 que contiene: 20\% arracacha, 20\% achira, 15\% mashua, 15\% oca, 20\% cañihua y 20\% tarwi, influye significativamente en la ganancia de peso y talla, el cual permite el diseño de productos alimentarios con valor agregados.

\section{RECOMENDACIONES}

En el ámbito de la lucha contra la desnutrición, y combate de la pobreza, las implicancias directas en la formulación de políticas públicas, principalmente, se debe fomentar el uso de formulaciones de alimentos con base a cultivos andinos, tales como arracacha (Ar) Arracacia xanthorrhiza, achira (Ac) Canna indica L., mashua (Ma) Tropaeolum tuberosum, oca (Oc) Oxalis tuberosa, cañihua (Ca) Chenopodium pallidiulle aellen, tarwi (Ta) Lupinus mutabilis, no solo por su importancia calórica y proteica, sino por sus aportes nutricionales en la ganancia de peso y talla. En ese sentido, se recomienda, que los programas sociales como: desayuno escolar, programas de educación y salud, que estén articulados, para conseguir un efecto directo en la generación de empleo e ingreso en las economías familiares agrarias, y de ese modo, construir una estrategia del tipo "win to win" con efectos directos en las economías regionales del país.

\section{REFERENCIAS BIBLIOGRÁFICAS}

Alpizar Vergara, M., \& RuizWeber, A. (2013). Efectos de una dieta elevada en hidratos de carbono y lípidos, sobre el sistema inmunitario del intestino delgado en ratones Balb/c. Tesis para obtener el título de Licenciado en Nutrición. Toluca, México: Facultad de Medicina. Universidad Autonóma del Estado de México. Recuperado el 12 de Agosto de 2017, de http://ri.uaemex.mx/bitstream/ handle/20.500.11799/14350/407325. $p d f$ ? $s$ e $q$ u e $n c$ e $=1$

Apaza Ahumada, M. G. (2019). Efecto del consumo de cultivos andinos quinua, cañihua y tarwi sobre el incremento de peso y nitrógeno retenido en ratas Wistar. Journal of High Andean Research, 21(3), 194-204. DOI: http://dx.doi.org/10.18271/ria.2019.477.

Barrera, V., Tapia, C., \& Monteros, Á. (2004). Raíces y Tubérculos Andinos: Alternativas para la conservación y uso sostenible en el Ecuador. Serie: Conservación y uso de la biodiversidad de raíces y tubérculos 
andinos: Una década de investigación para el desarrollo (1993-2003). Quito-Lima, Ecuador-Perú: Instituto Nacional Autónomo de Investigaciones Agropecuarias, Centro Internacional de la Papa, Agencia Suiza para el Desarrollo y la Cooperación. Recuperado el 11 de Agosto de 2017, de http://www.iniap.gob.ec/nsite/images/ documentos/Ra\%C3\%ADces\%20 y \% 20 Tub \% C $3 \%$ A 9 rculos $\% 20$ Alternativas\%20para\%20el\%20uso\%20 sostenible\%20en\%20Ecuador.pdf

Barrientos Alvarado, C., Becerra Reséndiz, V., Flores Gómez, D., \& Beltrán García, X. (2013). El calcio minimiza la absorción de grasas en ratas. Hospital Juarez México, 80(4), 219-223. Recuperado el 22 de Octubre de 2017, de http://www.medigraphic. com/pdfs/juarez/ju-2013/ju134b.pdf

Carvajal-Larenas, F., Linnemann, A., Nout, M., Koziol, M., \& van Boekel, M. (2016). Lupinus mutabilis: Composition, Uses, Toxicology, and Debittering, Critical. Reviews in Food Science and Nutrition, 1454-1487. DOI:10.1080/10408398.2013.772089

Castañeda, B., Manrique, R., Gamarra, F., Muñoz, A., \& Lizaraso, F. (2008). Probiótico elaborado en base a las semillas de Lupinus mutabilis Sweet (chocho or tarwi) seeds. Acta Médica Peruana, 25, 210-215. Disponible en: http:// www.redalyc.org/articulo.oa?id=96625405

Cedeño Noblecilla, E. L. (2013). Formulación, Elaboración y Control de Calidad de un Alimento Balanceado para Ratones de Experimentación (Mus musculus) del Bioterio de la Escuela de Bioquímica y Farmacia de la Espoch. Riobamba, Ecuador. Disponible en: http://dspace.espoch.edu.ec/ bitstream/123456789/2691/1/56T00394.pdf

Chirinos-Arias, M. (2015). Andean Lupin (Lupinus mutabilisSweet)aplantwith nutraceuticaland medicinal potential. Revista Bio Ciencias, 3(3), 163-172. Disponible en: http://editorial.uan. edu.mx/BIOCIENCIAS/article/view/139/185
Cuj, M., Mazariegos, M., Pérez, W., Fischer, E., \& Román, A. (2017). Determinación de la ganancia de peso, calidad proteica y digestibilidad de ocho dietas a base de dos leguminosas, maní (Arachis hypogaea L.) y ajonjolí (Sesamum indicum L.) en ratas Wistar. Revista científica, 27(1), 2131. Disponible en: https://dialnet.unirioja. es/servlet/articulo?codigo $=6185770$

Fuentes Paredes, F. d., Mendoza Yanavilca, R. A., Rosales Fernández, A. L., \& Cisneros Tarmeño, R. A. (2008). Guía de manejo y cuidado de animales de laboratorio: ratón. Lima, Perú: Ministero de Salud- Instituto Nacional de Salud. Disponible en: http:// bvs.minsa.gob.pe/local/INS/962_INS68.pdf

Gallego-Villa, D. Y., Russo, L., Kerbab, K., Landi, M., \& Rastrelli, L. (2014). Chemical and nutritional characterization of Chenopodium pallidicaule (cañihua) and Chenopodium quinoa (quinoa) seeds. Emirates Journal of Food \& Agriculture, 609-615. DOI:10.9755/ejfa.v26i7.18187

García, W., \& Cadima, X. (2003). Manejo sostenible de la agrobiodiversidad de tubérculos andinos: Síntesis de investigaciones y experiencias en Bolivia. Conservación y uso de la biodiversidad de raíces y tubérculos andinas: Una década de investigación para el desarrollo (1993-2003). Cochabamba, Bolivia: Fundación para la Promoción y la Investigación de Productos Andinos (PROINPA), Alcaldía de Colomi, Centro Internacional de la Papa (CIP) Agencia Suiza para el Desarrollo y la Cooperación (COSUDE). Disponible en: http://www. proinpa.org/tic/pdf/Tuberculos $\% 20$ andinos/Tuberculos\%20andinos/pdf39.pdf

Gómez, G., Vargas, R., \& Quesada, S. (1998). Crecimiento y conversión alimenticia de ratas Sprage-dawley sometidas a la ingesta de extractos acuosos de pejibaye (Bactris gasipaes). Agronomía Costarricense, 22(2), 185-189. Recuperado 
el 15 de Octubre de 2017, de http:// www.mag.go.cr/rev_agr/v22n02_185.pdf

Instituto Nacional de Salud. (2012). Reglamento del Comité Institucional de Ética para el uso de Animales en Investigación $N^{\circ}$ 114-2012-J-OPE/INS. Lima, Perú: Comité Institucional de ética para el uso de animales en investigación. Disponible en: https:// cdn.www.gob.pe/uploads/document/ file/1205668/RJ.N\%C2\%BA_114-2012.pdf

Jacobsen, S., \& Mujica, A. (2006). El tarwi (Lupinus mutabilis Sweet.) y sus parientes silvestres. Botánica Económica de Los Andes Centrales, 458-482. Disponible en: https:// beisa.au.dk/Publications/BEISA\%20 Book\%20pdfer/Capitulo\%2028.pdf

León, A., \& Urbina, K. (2015). Formulación, evaluación nutricional y sensorial del pan de molde integral enriquecido con quinua (chenopodlum quinoa), cañihua (chenopodium pallidicaule) y chia (salvia hispánica I.). Tesis de pregrado. Nuevo Chimbote, Perú: Universidad Nacional del Santa.

Márquez-Montes, R., Altúzar-Carpio,L.I.,VillanuevaCarrillo, G., \& Palacios-Pola, G. (2010). Evaluación biológica de alimentos nutricionalmente mejorados en ratas Wistar. Lacandonia, 4(2), 53-61.

Martínez Haaker, M. E., \& Matallana Valdivia, M. F. (2013). Efecto de dietas con distinto contenido de fibra en el peso y grasa corporal en ratones BALB/C inducidos a obesidad. Tesis para optar por el grado de Licenciado en Nutrición y Dietética. Lima: Facultad de Ciencias de la Salud. Universidad de Ciencias Aplicadas.

Matos-Chamorro, A., \& Muñoz- Alegre, K. (2010). Elaboración de Pan con Sustitución Parcial de Harina Pre Cocida de Nuña (Phaseoleus vulgaris L.) y Tarwi (Lupinus mutabilis). Revista de Investigación en Ciencia y Tecnología de Alimentos, 1(1),31-35. Recuperado el 2020 de octubre de 20, de https://revistas.upeu.edu. pe/index.php/ri_alimentos/article/view/816

Mosquera Perea, D. E. (2014). Evaluación de la calidad de pellets extruidos elaborados con materias primas no convencionales para la alimentación de cerdos. Palmira, Colombia: Tesis para optar el grado de Maestría en Ingeniería Agroindustrial. Facultad de Ingeniería Administración. Universidad Nacional de Colombia. Recuperado el 20 de Setiembre de 2017, de http://www.bdigital.unal.edu.co/48701/1/ Dissa_Enith_Mosquera_Perea.pdf

Olza Meneses, J., Porres Foulquie, J., Urbano Valero, G., Martinez de Victoria, E., \& Gil Hernandez, A. (2008). Evaluación biológica de la calidad de una mezcla de proteínas para uso en nutrición enteral. Nutrición Hospitalaria, 23(3), 206-211. Disponible en: https://www. redalyc.org/pdf/3092/309226726015.pdf

Ortega-David, E., Rodriguez, A., David, A., \& Zamora-Burbano, A. (2010). Caracterización de semillas de Lupinus mutabilis sembrado en los andes de Colombia. Acta agronómica, 59, 111-118. Obtenido de http://www.bdigital.unal.edu. co/18305/2/14094-42145-1-PB.pdf

Pérez, G. (2007). Fundamentos del trabajo con animales de laboratorio en proyectos de investigación. Revista del Hospital J. M Ramos Mejía, 12(3).

Taguchi, G., Elsayed, A., \& Hsiang, T. (1989). Quality engineering in production system. New York, EUA: McGraw-Hill, Inc.

Tapia, M. E., \& Fries, A. M. (2007). Guía de campo de cultivos andinos. Lima, Perú: FAO y ANPE. Disponible en: http://www. fao.org/3/ai $185 \mathrm{~s} / \mathrm{ai} 185 \mathrm{~s} 00 . \mathrm{htm}$

Tapia, Miguel. (2000). Cultivos andinos Sub-expplotados y su aporte a la alimentación. Santiago de Chile. 